\title{
TERES
}

\section{Tail Event Risk Expectile based Shortfall}

\author{
Philipp Gschöpf* \\ Wolfgang Karl Härdle* \\ Andrija Mihoci*
}

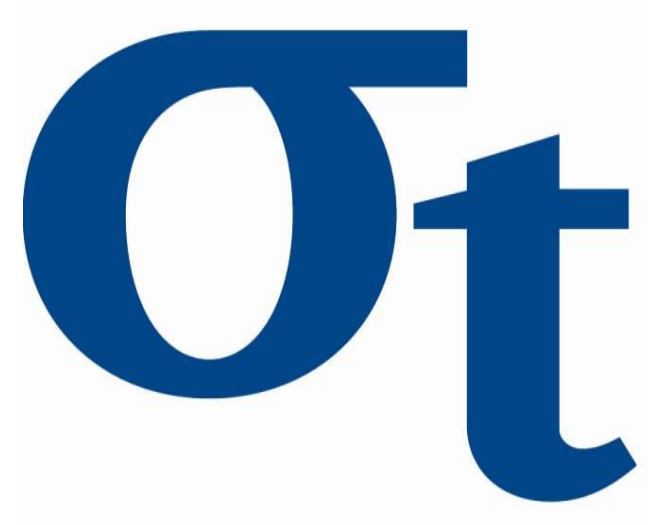

$\frac{v}{\sim}$

This research was supported by the Deutsche Forschungsgemeinschaft through the SFB 649 "Economic Risk". 


\title{
TERES - Tail Event Risk Expectile based Shortfall*
}

\author{
Philipp Gschöpfł, Wolfgang Karl Härdleªnd Andrija Mihoci ${ }^{\ddagger}$
}

August 28, 2015

\begin{abstract}
A flexible framework for the analysis of tail events is proposed. The framework contains tail moment measures that allow for Expected Shortfall (ES) estimation. Connecting the implied tail thickness of a family of distributions with the quantile and expectile estimation, a platform for risk assessment is provided. ES and implications for tail events under different distributional scenarios are investigated, particularly we discuss the implications of increased tail risk for mixture distributions. Empirical results from the US, German and UK stock markets, as well as for the selected currencies indicate that ES can be successfully estimated on a daily basis using a one-year time horizon across different risk levels.
\end{abstract}

JEL classification: C13, C16, G20, G28

Keywords: Expected Shortfall, expectiles, tail risk, risk management, tail events, tail moments

*Financial support from the Deutsche Forschungsgemeinschaft via CRC 649 "Economic Risk" and IRTG 1792 "High Dimensional Non Stationary Time Series", Humboldt-Universität zu Berlin, is gratefully acknowledged.

${ }^{\dagger}$ Humboldt-Universität zu Berlin, C.A.S.E. - Center for Applied Statistics and Economics, Spandauer Str. 1, 10178 Berlin, Germany, tel: +49 (0)30 2093-5703, fax: +49 (0)30 2093-5649, E-Mail: gschoepp@hu-berlin.de

${ }^{\ddagger}$ Humboldt-Universität zu Berlin, C.A.S.E. - Center for Applied Statistics and Economics, Spandauer Str. 1, 10178 Berlin, Germany and SKBI School of Business, Singapore Management University, 50 Stamford Road, Singapore 178899, tel: +49 (0)30 2093-5630, E-Mail: haerdle@hu-berlin.de

${ }^{\S}$ Humboldt-Universität zu Berlin, C.A.S.E. - Center for Applied Statistics and Economics, Spandauer Str. 1, 10178 Berlin, Germany, tel: +49 (0)30 2093 5728, fax: +49 (0)30 2093 5649, E-Mail: mihociax@cms.hu-berlin.de 


\section{Introduction}

Understanding of financial risk plays an important role in risk management and statistics. Researchers and practitioners aim to control financial distress by quantifying the uncertainty and risk dynamics through risk measures. Portfolios are subject to tail risk exposure, thus capturing the uncertainty stemming from extreme events is important in risk management. Since the dawn of statistical research, it has been documented that quantiles cannot successfully capture tail structures, Laplace (1793), nor is the methodological reasoning for using quantiles convincing, see e.g. Embrechts et al. (2005), Franke et al. (2015).

Expected Shortfall (ES) is a coherent risk measure, Artzner et al. (1998), Acerbi et al. (2001) discuss and quantify this risk exposure. This is contrary to the usage of a single Tail Event (TE), for example the tail probability (quantile) based Value at Risk (VaR), as ES takes diversification and risk aggregation effects properly into account, Delbaen (2002). ES is defined as the expected value of a random (return) variable below a given threshold. Its unique combination of desirable properties, Kusuoka (2001), has been recognized in portfolio selection, Bassett et al. (2004), and risk management. Recently ES has been recommended by the Basel Committee on Banking Supervision (2013) for internal usage and there is supportive evidence that it allows fund managers to comply with investor preferences more accurately compared to the VaR or variance based risk measures, Koenker (2005).

Tail Event Risk Expectile based Shortfall (TERES) offers tail-tailored ES. For given tail distribution the ES is provided through the concept of Generalized Tail Events (GTEs), Breckling and Chambers (1988), which helps us to analyze the ES variation stemming from the change of the underlying distributional framework. For example, the effect of 'lengthening or shortening the distribution tail' on ES can be studied by employing the normal-Laplace mixture. Our approach essentially quantifies the consequences of potential model misspecifications and biased risk indications in risk management that 
may result from the lack of tail-relevant observations, see, e.g., McNeil and Frey (2000). Concerning the robustness properties of ES, we focus on the effects of varying tail structures. A common argument for the usage of $\mathrm{VaR}$ is its low degree of sensitivity with respect to the tail structure change. Per contra, Stahl et al. (2012) advocate the usage of a more appropriate robustness notion and argue that, considering risk management applications, ES represents a robust risk measure. The Gaussian framework may only be suitable as long as the interest is on modeling the distributional center of a financial return time series, e.g., Hull and White (1998). Specifically for a downside tail risk measure, Acerbi and Tasche (2002), it is fundamental to be aware of the implications of deviations from the theoretical normal model on the resulting risk measures. TERES makes use of the normal-Laplace contaminated mixture, Huber (1964), to gain a better understanding of ES tail risk sensitivity.

The TERES framework is here applied to equity and foreign exchange market examples. Our research questions include: What are the thrills for ES estimation? How do the statistical characteristics of ES change across different distributions? Which risk range of ES is expected under different tail scenarios? In ES estimation we distinguish between two ES components, namely VaR and a correction term. This yields a more detailed description of investigated ES properties. The departure from the normality assumption furthermore implies a varying range for the risk measures under consideration. TERES displays that ES exhibits robustness qualities comparable to quantiles across selected distributional neighborhoods, a fact supported by empirical evidence from financial time series.

The paper is structured as follows. After considering tail event risk examples in Section 2. the TERES framework is introduced in Section 3. Empirical results concerning the ES analysis are provided in Section 4, whereas Section 5 concludes. 


\section{Tail Event Risk Examples}

Financial markets are characterized by time changing risk structures, and thus the uncertainty measurement associated with extreme Tail Events (TEs) has to be carefully treated. In this section we correspondingly discuss three examples: equity risk, foreign exchange exposure and portfolio allocation risk. After introducing tail risk characteristics for each example, associated risk measure dynamics and implications thereof are discussed. The risk assessment for the introduced return time series using TERES is provided in Section 4. In our paper, tail event risk modeling accounts for the (here) reported stylized facts, tail dynamics and observed tail events.

Example 1 (Equity risk) Equity returns exhibit non stationarity in the variance and higher order moments, most prominently in the kurtosis which serves as a TE indicator. At first glimpse such data may appear normally distributed, however even after timedynamic mean-variance standardization heavy tails prevail. Figure 1 displays QQ plots of DAX 30, FTSE 100 and S\&P 500 daily returns from 3rd January 2005 to 31st December 2014 (2608 trading days), standardized using a $\operatorname{GARCH}(1,1)$ model by Engle (1982) and Bollerslev (1986), against standard normal quantiles. The center of the data distribution may be well modeled using a conditional Gaussian setup. A clear "S" shape is nevertheless observed which cannot be successfully captured by conventional risk measures (e.g., VaR) that only consider tail event probabilities.

DAX

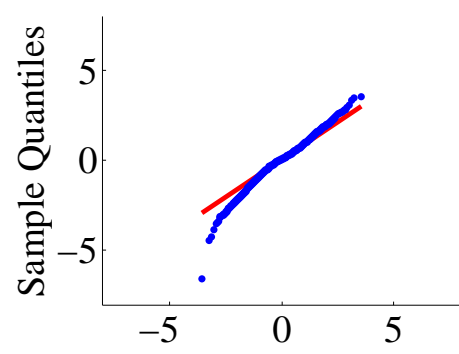

Normal Quantiles
FTSE 100

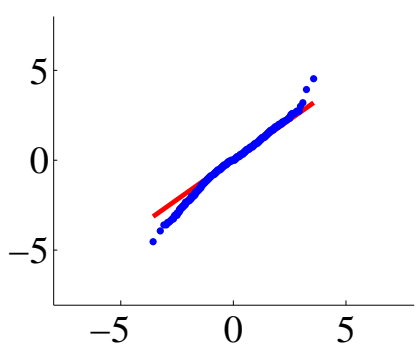

Normal Quantiles
S\&P 500

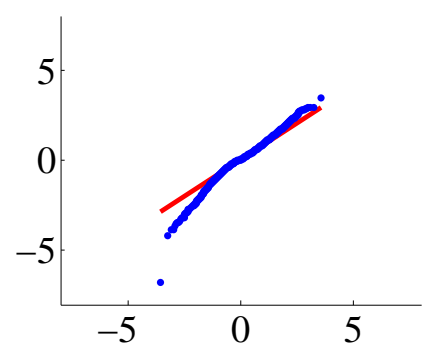

Normal Quantiles

Figure 1: QQ plots of standard normal quantiles and standardized daily return quantiles (blue dots) for DAX, FTSE 100 and S\&P 500 indices from 3rd of January 2005 to 31st of December 2014. The red line depicts the $45^{\circ}$ line.

Q TERES Standardization 
Example 2 (Foreign exchange exposure) Foreign exchange time series share similar features to those observed on stock markets. Consider the Euro (EUR) to Ukrainian Hryvnia (UAH) exchange rate, which inhibits two market distress periods: the financial crisis in 2008 and the political crisis in Crimea 2014/15. Figure 2 displays the observed and the standardized foreign exchange EUR/UAH returns from 3rd of January 2005 to the 1st of June 2015 (2715 trading days). The tail structure appears dynamic and even for the standardized time series non-normal; this is again an indication of non-stationarity in higher order moments, e.g., the kurtosis.

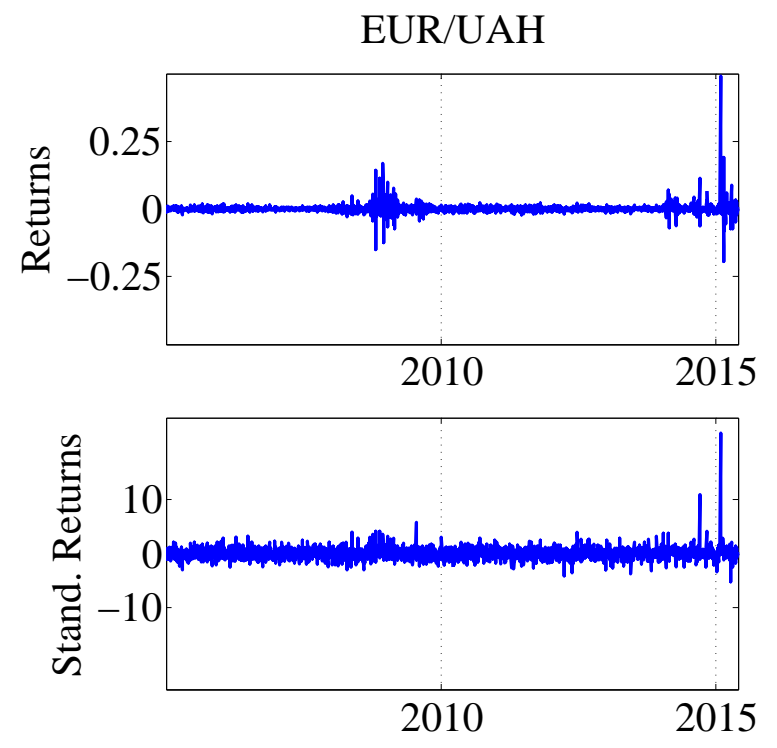

Figure 2: Returns and standardized returns using a $\operatorname{GARCH}(1,1)$ model for the EUR/UAH exchange rate from the 3rd of January 2005 to the 1st of June 2015.

Q TERES Standardization

Example 3 (Portfolio allocation risk) A successful asset allocation takes the tail structure into account. Consider for illustration the Tail Event Driven ASset allocation (TEDAS) framework by Härdle et al. (2014). Generally speaking, TEDAS enables to broaden the classical allocation by using quantile regression between the index series and portfolio constituents at selected fixed tail levels (e.g., 5\%, 15\%, 25\%, 35\% and $50 \%$ ). TEDAS allows to construct bespoke, TE driven portfolios. Returns of two TEDAS applications that significantly outperform the underlying index series are displayed in Figure 2 , 
(i) German equity market - TE weekly portfolios are formed out of a pool of 125 selected small and mid cap stocks for the span from 21st February 2014 to 28th November 2014 (41 trading weeks),

(ii) Worldwide mutual funds market - TE monthly portfolios are considered from 1st February 2008 to 31 January 2013 (73 trading months); here 583 mutual funds have been selected,

for details we refer to Härdle et al. (2015). As frequently encountered in risk management, both cases here result in relatively short time series due to weekly or monthly rebalancing schemes. Substantial amount of information about the underlying risk processes is nevertheless demanded in practice; this effect is intensified by the risk assessment requirement to accurately analyze the extreme outer tail. As explained in the sequel, TERES offers a suitable framework for evaluating (infrequently re-constructed) TE driven allocation strategies.
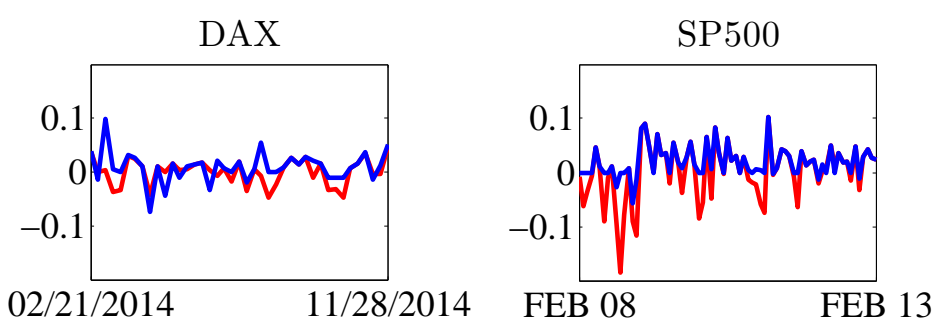

Figure 3: Returns of two TEDAS applications (blue) and their respective benchmarks (red). Left: weekly German stock portfolios vs DAX 30 index (41 trading weeks), right: monthly mutual funds portfolio returns vs S\&P 500 index (73 trading months)

Q TEDASstrategies2

\section{Tail Event Risk Expectile based Shortfall (TERES)}

There is general consensus about the advantageousness of ES over VaR as a measure of risk. TERES offers a flexible framework for examining the robustness properties of ES across different distributions and respective tail structures. Based on GTEs we identify ES components which are relevant for the inclusion of tail events to support the 
implementation of distributional shapes.

The ES of a r.v. $Y$ with cumulative distribution function (cdf) $F$ and probability density function (pdf) $f$ is defined as

$$
s_{\eta}=\mathrm{E}[Y \mid Y<\eta]
$$

with $\eta$ representing a given threshold. ES represents a coherent downside risk measure and with the threshold selected as the $\tau$-th quantile

$$
q_{\tau}=\inf _{y}\{y \mid \tau \leq F(y)\}
$$

we obtain a member of the class of spectral risk measures, Overbeck $(2004)$

It is important to note that - counter-intuitively to (1) - ES does not necessarily lead to increased margin requirements. We advocate to calibrate the risk level $\tau$ to ensure that coherent measurement of the portfolio risk does not necessitate increased expenses. The integration of tail perspectives into ES, on the other hand, is a more arduous issue. The concept of GTEs provides a distributional structure indicator that facilitates a connection between ES, VaR and tail scenarios.

\subsection{Generalized Tail Events and ES Decomposition}

A generalization of tail events is given by the concept of M-quantiles, Breckling and Chambers (1988). Specifically the quantile and the expectile denote two potentially different parameters of the more general M-quantile location model for a random variable (rv) $Y$, that is $Y=\theta+\varepsilon$. Given the M-quantile check function

$$
\rho_{\tau, \gamma}(u)=|\tau-\mathbf{I}\{u<0\}||u|^{\gamma}
$$


the $\tau$-th quantile and the $\tau$-th expectile respectively are identified as location parameter $\theta$ :

$$
\begin{aligned}
& q_{\tau}=\arg \min _{\theta} \mathrm{E}\left[\rho_{\tau, 1}(Y-\theta)\right] \\
& e_{\tau}=\arg \min _{\theta} \mathrm{E}\left[\rho_{\tau, 2}(Y-\theta)\right] .
\end{aligned}
$$

In terms of a location model, one sees that quantiles take solely the sign of residuals into account, while expectiles consider their magnitude.

Known M-quantile properties can be transferred onto ES. For this purpose, consider the expectile $e_{w_{\tau}}$ that equals the $\tau$-th quantile, i.e. $q_{\tau}=e_{w_{\tau}}$. Relating the expectile and the quantile by their corresponding levels $w_{\tau}$ and $\tau$ allows us to recover an indicator of the average distance of the tail probability mass from the quantile threshold $q_{\tau}$. Knowledge of the expectile level $w_{\tau}$ enables us to partition ES as follows

$$
s_{q_{\tau}}=s_{e_{w_{\tau}}}=e_{w_{\tau}}+\frac{e_{w_{\tau}}-\mathrm{E}[Y]}{1-2 w_{\tau}} \frac{w_{\tau}}{\tau}
$$

see, e.g., Taylor (2008). The resulting ES specification $s_{q_{\tau}}$ can be seen a function of the

expectile (or VaR) level, weighted by the ratio $\frac{w_{\tau}}{\tau}\left(1-2 w_{\tau}\right)^{-1}$. Among others, TERES is used to confirm whether 'lengthening the distributional tail' leads to an increase in the considered ratio, and furthermore, it serves as an instrument to compare the VaR and ES properties.

\subsection{Expectile Quantile Transformation and Tail Structures}

The characteristics of $w_{\tau}$ in the context of ES are scantly studied. If the underlying distribution $F$ is known, the Expectile Quantile Transformation (EQT) is given by

$$
w_{\tau}=\frac{L P M\left(q_{\tau}\right)-q_{\tau} \tau}{2\left\{L P M\left(q_{\tau}\right)-q_{\tau} \tau\right\}+q_{\tau}-\mathrm{E}[Y]}
$$


where

$$
\operatorname{LPM}(u)=\int_{-\infty}^{u} y f(y) d y
$$

denotes the lower partial moment. Expression $(7)$ holds for any $F$ due to the one-to-one mapping between expectiles and quantiles, Jones (1994). The Mass Distance Indicator (MDI) $\frac{w_{\tau}}{\tau}$ quantifies the average distance of the probability mass of $f$ from the $\tau$-quantile, which in TERES is utilized in tail focused inference.

The EQT (7) depends on the underlying distributional specification. In order to shed some light on this let us consider four cases: (a) uniform, (b) standard normal, (c) Laplace and (d) stable EQT.

(a) Let $Y$ denote a standard uniform distributed variable $Y \sim U[0,1], f(y)=\mathbf{I}\{0 \leq y \leq$ $1\}$ with $q_{\tau}=\tau$. The EQT level equals

$$
w_{\tau}=\frac{\tau^{2}}{2\left(\tau^{2}-\tau+0.5\right)}
$$

Graphical inspection of (9) reveals that in this case the $\tau$-expectile is closer to the distributional center than the $\tau$-quantile.

(b) As discussed above, industry relevant case of misspecification oftentimes occur when a normality assumption is applied to heavy-tailed data. For a standard Normal distribution the LPM is equal to $-\varphi(u)$ and therefore

$$
w(\tau)=\frac{-\varphi\left(q_{\tau}\right)-q_{\tau} \tau}{-2\left\{\varphi\left(q_{\tau}\right)+q_{\tau} \tau\right\}+q_{\tau}-\mathrm{E}[Y]}
$$

Again we find the expectile to be closer to the distributional center, see Figure 4. 


\section{Quantiles vs. Expectiles}

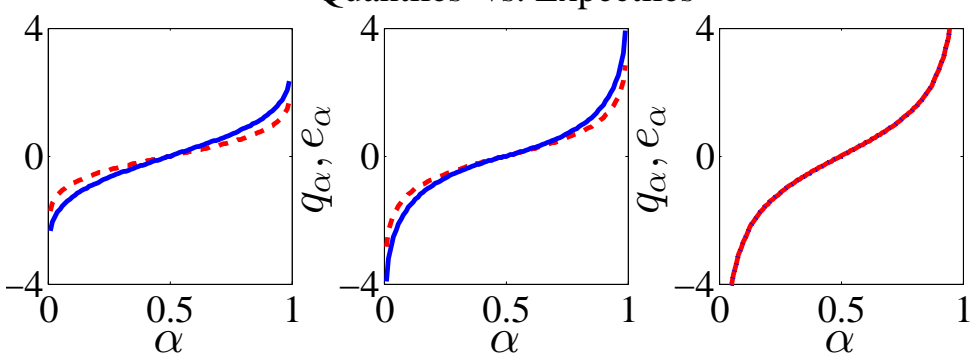

Difference

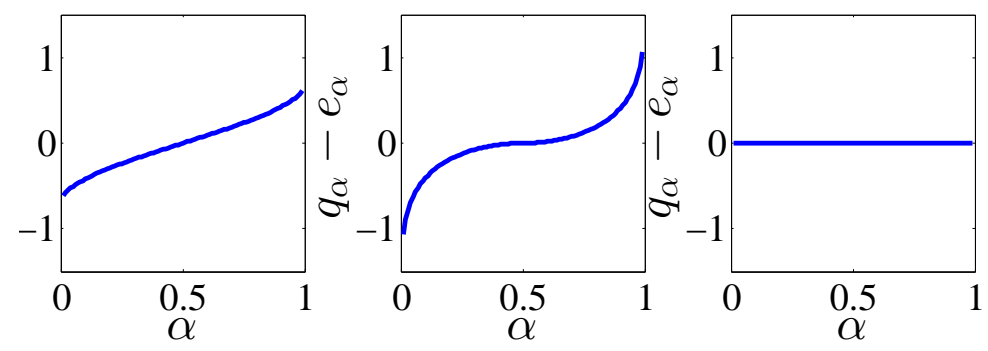

Figure 4: Top: Quantiles (blue) and Expectiles (red) and differences thereof (bottom) for 3 cases: standard normal (left), standardized Laplace (middle) and the cdf given in (11) (right)

Q TERES_ExpectileQuantileDiffMulti

(c) For the slightly heavier-tailed standardized Laplace (i.e. double exponential) distribution the LPM corresponds to

$$
L P M(u)= \begin{cases}\exp (u)(u-1), & \text { if } u<0 \\ \exp (-u)(-u-1), & \text { else }\end{cases}
$$

which is characterized by a more pronounced " $\mathrm{S}$ " shape than the normal case, as displayed in Figure 4. On average the MDI $\frac{w_{\tau}}{\tau}$ increases as the degree of tail heaviness is increased. The conclusion is less clear in the extreme outer tail though. Additionally we may ask: will the $\tau$-expectile generally be closer to the distributional center than the $\tau$-quantile? To provide answers we consider the distribution, Koenker (1993)

$$
F(x)= \begin{cases}0.5-0.5\left(1-\frac{4}{4+x^{2}}\right)^{0.5}, & \text { if } x<0 \\ 0.5+0.5\left(1-\frac{4}{4+x^{2}}\right)^{0.5}, & \text { else }\end{cases}
$$


which yields $e_{\tau}=q_{\tau}$ for $\tau \in[0,1]$. This distribution exhibits considerably heavier tails than typical distributions occurring in financial markets reality. In fact it exhibits such heavy tails that marginal increases of it's tail probability mass render the second moment undefined. Thus we conclude: for a realistic degree of tail heaviness $w_{\tau}<\tau$ holds for the lower tail. However, we wish to extend the analysis to a wider class of functions.

(d) The class of stable distributions, Mandelbrot (1963), Fama (1965) and Cizek et al. (2011), enables us to do so. It captures a wide array of pdfs (including normal and Cauchy), reflected by a stability index $\alpha \in(0,2]$ and a skewness parameter $\beta \in[-1,1]$. Location and scale are controlled for by $\mu$ and $\sigma$. A closed form solution for it's cdf does not exist. An approximation of the lower tail for this class of distributions is given by, Nolan (2015)

$$
\begin{aligned}
f(y \mid \alpha, \beta, \sigma) & \approx c_{\alpha} \sigma^{\alpha}|\beta-1|(-y)^{-\alpha-1} \\
c_{\alpha} & =\alpha \sin \left(\frac{\pi \alpha}{2}\right) \frac{\Gamma(\alpha)}{\pi}
\end{aligned}
$$

Therefore, for $\alpha \in[1,2]$, the LPM of stable distributions is approximated as

$$
L P M_{\alpha}(u) \approx \frac{c_{\alpha} \sigma^{\alpha}|\beta-1|}{1-\alpha}(-u)^{1-\alpha}, \quad \text { as } u \rightarrow-\infty
$$

For simplicity we consider the standardized symmetric case $(\sigma=1, \beta=0)$. The stability index $\alpha$ allows the controlled increase of the degree of tail-heaviness. As $\alpha$ increases the tail becomes lighter and the MDI is (again) found to decrease. Perhaps even more interestingly, the example points out the LPM as the parameter that is most dependent on the selection of the underlying $f$, especially in the extreme outer tail. Consider e.g. $q_{\tau}=-5$ where the LPM lies within the range $[6.23 \mathrm{e}-19,0.01]$ and the according $\tau$ in [0.0002, 0.04]. In other words: ES which depends on the LPM is less distributional robust than VaR.

The question remains - however - if we can quantify the sacrifice in robustness as market 
participants progress from VaR to ES. We will proceed with an analysis of the connection between the distributional (tail-) information provided by the MDI and the ES, thereby formulating the TERES framework that will allow us to give an answer.

\subsection{Tail Event Risk Modeling Framework}

The non-normality apparent on many time series renders the comparison the robustness properties of ES and VaR inside a realistic distributional neighborhood crucial to the understanding of model misspecification implications. Even though our focus here is on financial applications, this holds true for most statistical studies.

We here model the tail structure by adopting a range of distributions (scenarios). To analyze the robustness properties of ES under deviations from a normality assumption, we here follow the approach outlined in Huber (1964). The underlying idea is to emphasize the usage of a neighborhood for robustness analysis. The aim is to find an estimator, that displays good performance as one sticks loosely to an underlying model assumption, e.g. normality, but does rely on the theoretical model to be literally exactly true. We then continue by providing a detailed analysis of the maxima and minima risk indications within the selected distributional neighborhood.

The contaminated neighborhood for a r. v. $Y$ has pdf:

$$
f(y \mid \delta)=(1-\delta) \varphi_{\theta_{1}}(y)+\delta h_{\theta_{2}}(y)
$$

where $\varphi$ denotes a normal pdf with $\theta_{1}=\left(\mu, \sigma_{1}\right)^{\top}$

$$
\varphi_{\theta_{1}}(u)=\frac{1}{\sigma_{1} \sqrt{2 \pi}} \exp \left\{-\frac{(u-\mu)^{2}}{2 \sigma_{1}^{2}}\right\}
$$

and $h$ another continuous symmetric distribution with parameter set $\theta_{2}$. We now provide

a Maximum Likelihood (ML) based argument for the usage of Laplacian contamination 
in EQT analysis. Consider the probability density of an asymmetric Generalized Error Distribution (GED) given as, Ayebo and Kozubowski (2003)

$$
f(x)=\frac{\gamma}{\sigma \Gamma\left(\frac{1}{\gamma}\right)} \frac{\kappa}{1+\kappa^{2}} \exp \left\{\left(-\frac{\kappa^{\gamma}}{\sigma^{\gamma}} \mathbf{I}\{x-\mu \geq 0\}-\frac{1}{\kappa^{\gamma} \sigma^{\gamma}} \mathbf{I}\{x-\mu<0\}\right)|x-\mu|^{\gamma}\right\}
$$

With the gamma function $\Gamma(x)=\int_{0}^{\infty} x^{t-1} \exp (-x) d x . \sigma$ is the scale parameter, $\gamma$ determines the shape and $\kappa$ the degree of skewness. $\mu$ is the location parameter. For simplicity let $c(\gamma, \sigma, \kappa)=-\log \frac{\gamma}{\sigma \Gamma\left(\frac{1}{\gamma}\right)} \frac{\kappa}{1+\kappa^{2}}$. The log-likelihood of the asymmetric GED is

$$
-\log \{f(x \mid \mu, \sigma, \gamma, \kappa)\}=c(\gamma, \sigma, \kappa)+\left(\frac{\kappa^{\gamma}}{\sigma^{\gamma}} \mathbf{I}\{x-\mu \geq 0\}+\frac{1}{\kappa^{\gamma} \sigma^{\gamma}} \mathbf{I}\{x-\mu<0\}\right)|x-\mu|^{\gamma}
$$

Comparison with the M-quantile check function (3) reveals that for a suitable choice of shape and skewness the resulting location MLE is equivalent to the M-quantile. This is achieved by selecting $\kappa$ as

$$
\kappa^{*}(\gamma)=\left(\frac{\tau}{1-\tau}\right)^{\frac{1}{2 \gamma}}
$$

For the asymmetric Laplace $(\gamma=1)$ and asymmetric normal $(\gamma=2)$ distributions the quantile estimator and the expectile result as location parameter estimate. Thus we propose the usage of Laplace contamination $h$ for the purpose of EQT analysis. The parameter subset is re-parametrized as $\theta_{2}$ is $\left(\mu, \sigma_{2}\right)^{\top}$ and

$$
h_{\theta_{2}}(u)=\frac{1}{\sigma_{2} \sqrt{2}} \exp \left\{-\sqrt{2} \frac{|u-\mu|}{\sigma_{2}}\right\}
$$

Now that a suitable distribution $h$ is at hand we present the distributional environment (15) in more detail. The most light tailed scenario results for $\delta=0$, where a normal distribution is always employed. For increasing values of $\delta$ the distributional tail is 
lengthened, until at $\delta=1$ the more heavy tailed Laplace distribution is selected with certainty. As we are interested in slight departures from the normality assumption our focus of attention will be on the neighborhood resulting from $\epsilon$ values that are close to 0 .

Obviously the expectile, quantile and the corresponding ES will vary within the selected neighborhood. Within a $\delta$-contaminated neighborhood as in $15, w_{\tau}$ becomes a function of $\delta$. In settings with contamination that exhibits heavier tails than under normality, such as Laplacian, this relationship is monotonically decreasing in $\delta$ for low risk levels (such as $\tau=0.05$ or $\tau=0.10$ ) if the ES is calculated strictly based only the assumed distribution. Our approach however makes use of (6) by combining pre-estimated quantiles or expectiles with the relevant expectile level $w_{\tau}$. This turns out to be specifically tailored to our needs, as we are interested in comparing the robustness properties of ES and $\mathrm{VaR}$ which originate in the influence of $w_{\tau}$. Thus we keep the $\tau$-quantile fixed while varying $w_{\tau}$ over the selected distributional neighborhood.

As an example consider the standardized Laplacian contamination case as defined in (20), (16) and (15) with parameter set $\left(\mu=0, \sigma_{1}=1, \sigma_{2}=1\right)^{\top}$. The upper left part of Figure 5 shows the resulting ES for levels of contamination $\delta$ ranging from 0 (normal) to 1 (Laplace case). To analyze the neighborhood around the normal scenario in the outer tail we provide the ES for the $\tau=0.005$ risk level in the top right part of Figure 5. We note that the strongest variation of the ES, i.e. the absolutely highest slope, is observed at the normal scenario. This is very reasonable, as Huber (1964) points out that lengthening the tail structure, starting from a light tailed case has a more pronounced influence on the estimator than a shortening of tails. Nevertheless, as (6) shows that knowledge of the quantile could potentially provide a dramatical reduction in ES variation. In financial examples we find this effect to be very strong, as displayed by empirical applications of TERES in section 4 . 

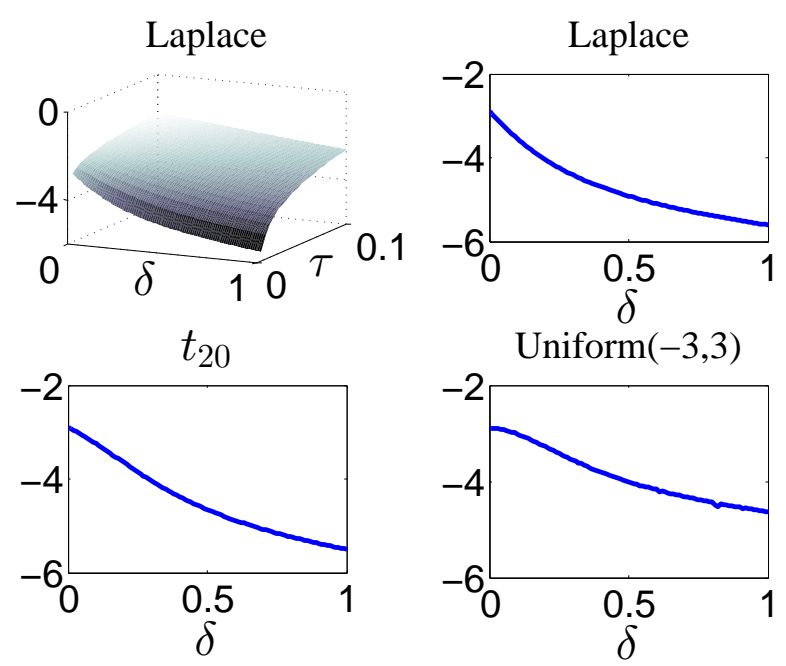

Figure 5: Theoretical $\mathrm{ES}_{q_{\tau}}$ for mixtures of standard Normal and standardized Laplace (top left). Furthermore, the Laplace, $t$ with $20 \mathrm{df}$ as well as Uniform(-3,3) contaminated normal environments of degree $\delta$ for the fixed (tail) risk level $\tau=0.5 \%$ are depicted.

Q TERES_ES_Analytical

Yet, before we progress to this empirical analysis, the sensitivity of the EQT with respect to the M-Quantile norm remains to be discussed. Regarding (3) it becomes apparent that (7) results as a special case for $\gamma=2$ and the question arises how strong the influence of the norm selection is here. In the given framework the norm is controlled by fixing a $\gamma \geq 1$. Note that

$$
w(\alpha, \gamma)=\frac{\int_{-\infty}^{q_{\alpha}}\left|y-q_{\alpha}\right|^{\gamma-1} d F(y)}{\int_{-\infty}^{\infty}\left|y-q_{\alpha}\right|^{\gamma-1} d F(y)}, \quad \gamma \geq 1
$$

holds. Therefore, for the special case of quantiles $w(\alpha, 1)=\alpha$. The analysis reduces to consider a value $\gamma>1$ (i.e. 2 for the expectile case) and to then investigate the convergence rate, with respect to $\gamma$, of the term $\left|y-q_{\alpha}\right|^{\gamma-1}$ towards one. Generally we thus observe exponential convergence for $\left|y-q_{\alpha}\right|>1$ whereas for $\left|y-q_{\alpha}\right|<1$ root convergence results. For $\left|y-q_{\alpha}\right|>1 \approx 1$ nearly linear convergence towards the quantile case is observed. Therefore we conclude that - using standardized data and modest risk - levels the framework shows a reasonable degree of stability in the selection of the loss function. 


\section{Empirical Study}

In this section TERES is employed to investigate the properties of ES given a time series of empirical quantile estimates. As introduced in Section 2, we demonstrate TERES for three stock indices, namely the DAX, the FTSE and the S\&P 500. Thereafter an FX rate example is presented using the EUR to Ukrainian Hryvnia (UAH) exchange rate which exhibits two noticeable periods of temporally increased tail-heaviness. The third example utilizes TERES for a pre-selected equity portfolio with time changing component weights.

\subsection{Equity Application}

Figure 6 displays the 1 percent VaR and ES for normal and Laplace scenarios for the selected equity indices. To emphasize the feasibility of TERES for small sample situations, estimation was carried out using a moving window approach with a window-size of 250 trading days. For each moving window the empirical quantile is calculated and a preselected scenario is then used to calculate the tail indicator $w_{\tau}$. The returns where rescaled using a GARCH(1,1) model and a constant mean. An ES estimate is obtained by rescaling with the estimated standard deviation. As the interest of this research lies in investigating the influence of the scenario selection we display the standardized results wherever they were applicable. 

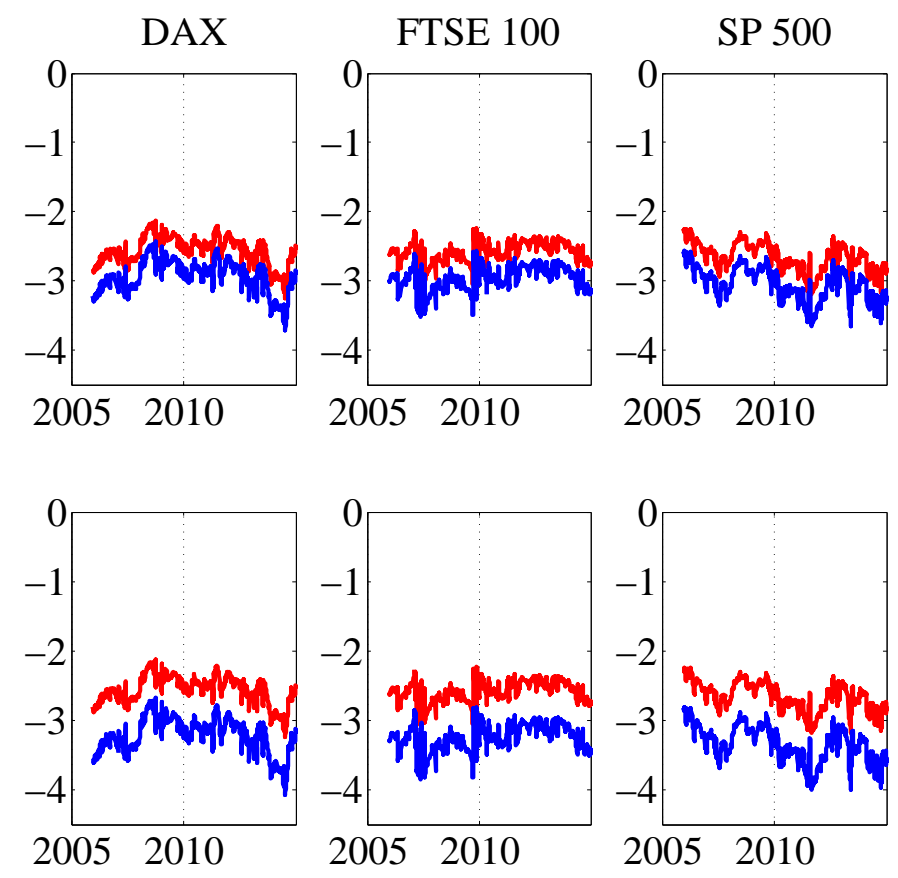

Figure 6: $s_{e_{w_{\tau}}}$ (blue) and corresponding VaR (red) using a rolling window of 250 days and a 1 percent risk level; normal scenario $(\delta=0)$ above and Laplace scenario $(\delta=1)$ below

Q TERES_RollingWindow

The variation of ES over the scenarios, is seen in the difference of the blue curves in the top and bottom sections of Figure 6. Percentage-wise this dependence is outweighed by the influence of the quantile (in red). After rescaling the effect becomes even less pronounced - percent wise - as the capturing of the heteroscedasticity of the original return series partially reflects the risk dynamics. This low influence of the distributional variations is indicating, that the TERES approach to ES estimation is exhibiting robustness properties comparable to VaR in financial market applications.

Since the empirical quantile was employed for estimation the ES the resulting surface is not ensured to be monotonously increasing in the degree of tail heaviness. Table 1 displays the ES for the last moving window, i. e. for the last 250 day sample, for varying degrees of tail-heaviness, here controlled by the contamination degree $\delta$. Lengthening the tail of the normal case has a much more pronounced effect than shortening the tail of the Laplace case, analog to the results in Huber (1964). 


\begin{tabular}{l|ccc}
\hline \hline \multicolumn{1}{c|}{$\delta$} & DAX & FTSE 100 & S\&P 500 \\
\hline 0.0 & -2.91 & -3.11 & -3.26 \\
0.001 & -2.91 & -3.11 & -3.26 \\
0.002 & -2.91 & -3.12 & -3.27 \\
0.005 & -2.92 & -3.13 & -3.28 \\
0.01 & -2.94 & -3.14 & -3.30 \\
0.02 & -2.97 & -3.17 & -3.33 \\
0.05 & -3.05 & -3.26 & -3.42 \\
0.1 & -3.16 & -3.38 & -3.54 \\
0.15 & -3.24 & -3.46 & -3.63 \\
0.25 & -3.32 & -3.55 & -3.72 \\
0.5 & -3.30 & -3.53 & -3.70 \\
1.0 & -3.19 & -3.41 & -3.57 \\
\hline \hline
\end{tabular}

Table 1: Estimated $s_{e_{w_{\tau}}}$ for selected indices at $\tau=0.01$, from from Jan. 16 to Dec. 31 2014 (250 trading days)

Q TERES_RollingWindow

As displayed in Table 2, the value of the contamination level $\delta$ that achieves the highest absolute risk indication is at $\frac{1}{3}$, independent of the underlying sample. In the selected case of a Laplacian contamination we find a high degree of robustness for the ratio of maximal and minimal risk indication. This results carries over to other realistic distributional neighborhoods and further motivates the usage of TERES as a tool for risk corridor construction. It further emphasizes the low influence of the risk scenarios, i. e. the parametric part of the estimation procedure and thus a high degree of robustness.

\begin{tabular}{l|ccc}
\hline \hline \multicolumn{1}{c|}{$\delta$} & DAX & FTSE 100 & S\&P 500 \\
\hline Maximal ES & -2.91 & -3.11 & -3.43 \\
Associated $\delta$ & 0.0 & 0.0 & 0.0 \\
& & & \\
Minimal ES & -3.33 & -3.57 & -3.97 \\
Associated $\delta$ & $\frac{1}{3}$ & $\frac{1}{3}$ & $\frac{1}{3}$ \\
& & & \\
ES variation & $14.6 \%$ & $14.6 \%$ & $14.6 \%$ \\
VaR & -2.54 & -2.72 & -2.99 \\
\hline \hline
\end{tabular}

Table 2: Maximal ES variation over a normal-Laplace mixture for standardized selected indices at the $q_{0.01}$ threshold, from Jan. 16 to Dec. 312014

Q TERES_Range 


\subsection{Exchange Rate Application}

As elaborated above, the EUR to UAH FX rate exhibited time dynamic tail structures, which poses further complications to the calculation of (extreme) tail risk measures for this time series.

Applying TERES directly to the non-standardized data, as depicted in Figure 7, allows a study of the effects of heteroscedasticity on the resulting ES estimate and it's robustness properties. The risk structure of the underlying time series as depicted in Figure 2 is captured and the distributional sensitivity is reasonable. The upper part of Figure 7 depicts the ES at the one-percent quantile for the lightest tail (normal distribution) in blue and for the worst case $\left(\delta \approx \frac{1}{3}\right)$ in green. The two ES dynamics thus form a corridor of the highest and lowest risk indication given the empirical quantile.

The two depicted cases to allow to emphasize the applicability of TERES in a setting where the investor fixes the tail shape up front (for example due to legal reasons). Even though the two scenarios (by definition) lie further apart than the normal and the Laplace scenario the risk corridor is comparably narrow, an observation that coincides with the results obtained in the other examples.

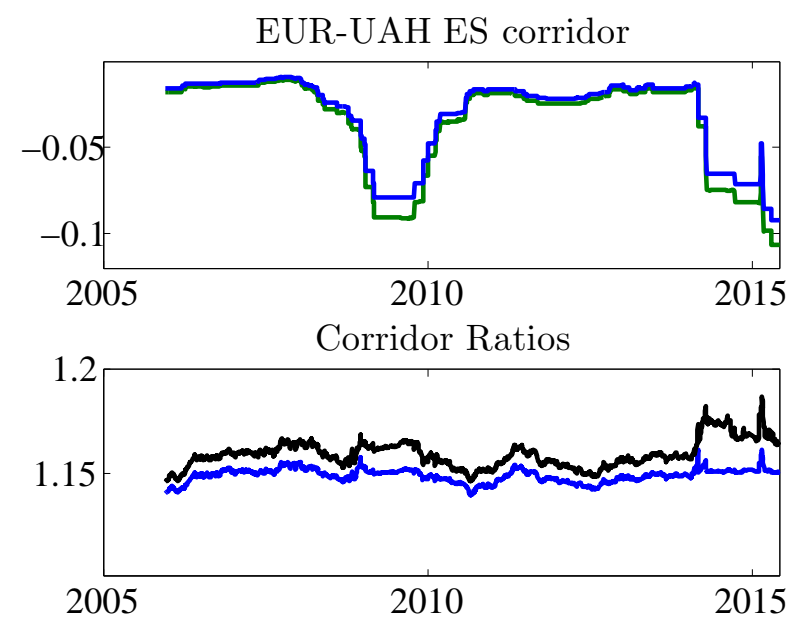

Figure 7: Top: $s_{q_{0.01}}$ using a rolling window of 250 days for TERES; normal scenario (blue) and high risk scenario (green). Bottom: Ratios of high risk and low risk scenario ES estimates for risk levels $\tau$ being 1 percent (blue) an 5 percent (black).

Q TERES_RollingWindow 
The lower part of Figure 7 depicts the ratio of the ES estimates under the corresponding lowest and highest (tail) risk indications for risk levels $\tau$ one and five percent. The risk corridor for the five percent risk level for the Laplace case was found as having contaminations of $\delta=0$ and $\delta=0.73$ for the lowest and highest risk scenarios respectively. The ratio, and therefore the variation of the ES over the scenarios, is seen to not exceed 1.20 (i.e. we find less than 20 percent additional variation as one progresses from VaR to ES) for both cases and is here even lower as one progresses towards the outer tail. During the two market distress scenarios in 2009 and 2015 the corridor bound ratios are displaying only minor increases and we find the 1 percent risk level ES to be much less affected than the 5 percent risk level ES.

\subsection{Equity Portfolio Application}

The tail optimized TEDAS return series introduced in Section 2 consist of 41 and 73 observations, respectively. This sparse availability of data makes it challenging to estimate the tail risk of the selected portfolio. However, the usage of expectiles allows to overcome numerous obstacles encountered in small sample situations. The top part of Figure 8 depicts the $\tau$-quantile and the resulting $\mathrm{ES}_{q_{\tau}}$ estimate for the TEDAS portfolio selected from DAX constituents. The usage of the quantile here not only causes the estimate to be non-smooth, it also induces non-monotonicity in the ES estimate. Usage of the empirical expectile delivers a more suitable estimate, as depicted in the bottom part of Figure 8 .

The depicted normal and tail-heavy (Laplace contamination with $\delta \approx \frac{1}{3}$ ) scenarios both show a considerably higher degree of smoothness with respect to variation in the risk level $\tau$. Comparison of the quantile and expectile based ES realizations under a normal scenario (both in blue), reveals that the expectile based method indicates higher risks than the quantile based estimates. Note that the empirical quantile employs linear interpolation, whereas the evidently exponential tail structure here is more appropriately captured by the expectile. 

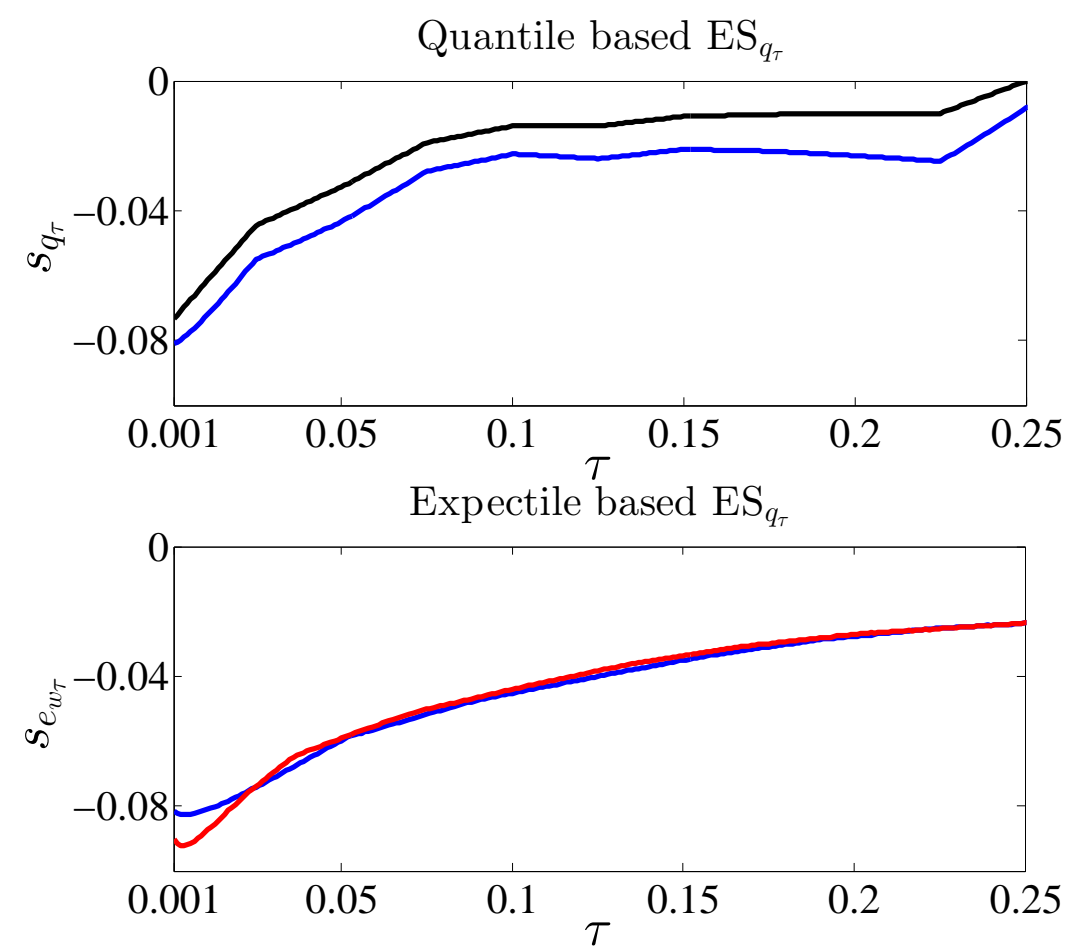

Figure 8: $s_{q_{\tau}}$ (i.e. $s_{e_{w_{\tau}}}$ ) for a TEDAS portfolio. Top: empirical quantile (black) and the resulting normal scenario ES (blue). Bottom: Expectile based ES, normal scenario (blue) and high-tail risk scenario (red)

The expectile based ES for the globally selected TEDAS portfolio is displayed in the lower part of Figure 8. Two scenarios are shown: Normal and the tail heavy $\left(\delta=\frac{1}{3}\right)$ Normal-Laplace scenario that results in the maximal risk indications at $\tau=0.01$. The sample here consists of 73 observations. For $0.0022 \leq \tau<0.5$ the scenarios deliver reasonable results, thereby demonstrating a strong performance capability for applied risk management. Afterwards the estimate degenerates, due to the limited support of the empirical expectile. As above, a low variation over the scenarios is observed which becomes less pronounced as one considers risk levels $\tau$ closer to the distributional center. 

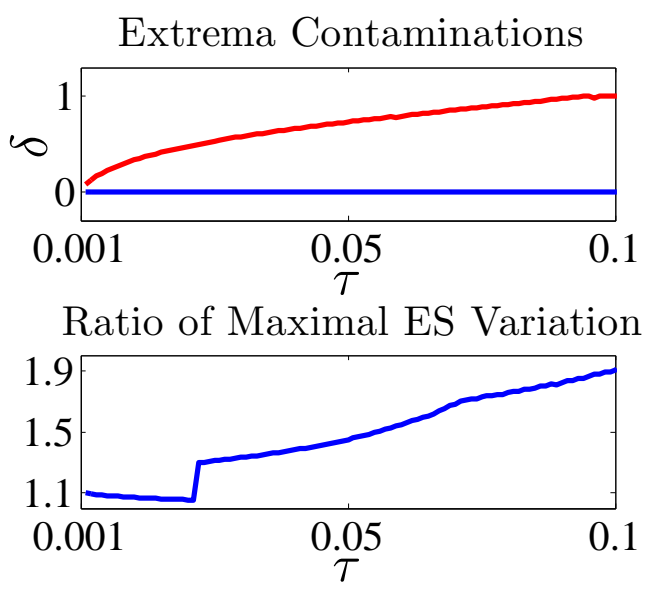

Figure 9: Top: contamination levels $\delta$ corresponding to the safest (blue) and riskiest (red) scenarios. Below: ratio of the absolute lowest and highest ES indications

Q TERES_Range_TEDAS

Further of interest are the properties of the risk corridor, i.e. the range from minimal to maximal ES indication inside a given distributional neighborhood. Figure 9 displays the contamination levels $\delta$ corresponding to the risk indication extrema for a NormalLaplace neighborhood. The ratio of the lowest and highest ES estimation for the TEDAS portfolio of global constituents is displayed in the lower part of the Figure. The ratio here increases for large risk levels as the maximal risk indication becomes closer to zero. Of more general importance though is the consideration of the outer lower tail, where it is apparent that the risk corridor displays a comparative narrowness for $\tau<0.022$ (i.e. where the high risk scenario is constituted by the Laplacian case). This especially indicates a high degree of distributional robustness for the outer tail.

The empirical section above presented applications over several asset classes of TERES. Firstly, it was found that ES displayed robustness properties comparable to VaR as the underlying distribution was varied. Secondly, evidence was presented that expectiles enable to achieve a more smooth ES estimation than quantiles, which was found to be especially advantageous in small sample situations. 


\section{Conclusions}

The TERES technique employs expectiles to estimate ES. The usage of expectiles allows to estimate ES more smoothly and reduces the estimation bias. The approach outlined here allows to consider ES in a full range of distributions. Furthermore we enable a more rigorous comparison of ES and VaR. The focus of this work is on examine the robustness properties of ES.

The main finding confirms recent results of, e.g., Bellini et al. (2014) that, considering realistic financial risk settings, ES has robustness properties that are comparable to VaR. We demonstrated that this enables the TERES approach to offer a combination of desirable properties, permitting it to cope with phenomena such as leptocurticity. At the same time expectile based TERES offers a suitable mean to tackle tail risk analysis and quantification under small samples.

The modular structure of TERES enables extension possibilities which enable new opportunities for research and application. A prominent example to consider is, e.g., the Expectation Maximization (EM) algorithm, Hartley and Rao 1967) and Dempster et al. (1977), which enables to determine the most likely scenario.

\section{References}

Acerbi, C., Nordio, C. and Sirtori, C. (2001). Expected shortfall as a tool for financial risk management, arXiv preprint cond-mat/0102304 .

Acerbi, C. and Tasche, D. (2002). On the coherence of expected shortfall, Journal of Banking \& Finance 26(7): 1487-1503.

Aigner, D., T., A. and Poirier, D. (1976). On the estimation of production frontiers: Maximum likelihood estimation of the parameters of a discontinuous density function, Journal of economic review $\mathbf{1 7}(2)$. 
Artzner, P., Delbaen, F., Eber, J. M. and Heath, D. (1998). Coherent measures of risk.

URL: http://www.math.ethz.ch/delbaen/ftp/preprints/CoherentMF.pdf

Ayebo, A. and Kozubowski, T. J. (2003). An asymmetric generalization of gaussian and laplace laws, Journal of Probability and Statistical Science 1(2): 187-210.

Basel Committee on Banking Supervision (2013). Fundamental review of the trading book: A revised market risk framework, Consultative Document .

Bassett, G. W., Koenker, R. and Kordas, G. (2004). Pessimistic portfolio allocation and choquet expected utility, Journal of Financial Econometrics 2(4): 477-492.

Bellini, F., Klar, B., Müller, A. and Gianin, E. (2014). Generalized quantiles as risk measures, Insurance: Mathematics and Economics 54(C): 41-48.

Bollerslev, T. (1986). Generalized autoregressive conditional heteroskedasticity, Journal of Econometrics 31(3): 307-327.

Breckling, J. and Chambers, R. (1988). M-quantiles, Biometrica 75(4).

Cizek, P., Härdle W. K. and Weron, R. (2011). Statistical Tools for Finance and Insurance. 2nd ed., Springer Verlag, Heidelberg. ISBN: 978-3-642-18061-3.

Delbaen, F. (2002). Coherent risk measures on general probability spaces, Advances in finance and stochastics : 1-37.

Dempster, A., Laird, N. and Rubin, D. (1977). Maximum Likelihood from Incomplete Data via the EM Algorithm, Journal of the Royal Statistical Society. Series B (Methodological) 39(1): 1-38.

Embrechts, P., Frey, R. and McNeil, A. (2005). Quantitative risk management, Princeton Series in Finance 10.

Engle, R. F. (1982). Autoregressive conditional heteroscedasticity with estimates of the variance of united kingdom inflation, Econometrica 50(4): 987-1008.

Fama, E. F. (1965). The behavior of stock-market prices, Journal of business : 34-105. 
Franke, J., Härdle, W. K. and Hafner, C. M. (2015). Statistics of Financial Markets: an Introduction. 4th ed., Springer Verlag, Berlin Heidelberg. ISBN: 978-3-642-54538-2, e-ISBN 978-3-642-54539-9 (555 p), DOI: 10.1007/978-3-642-54539-9.

Härdle, W. K., Nasekin, S., Lee, D. K. C., Ni, X. and Petukhina, A. (2015). Tail Event Driven ASset allocation: evidence from equity and mutual funds markets, Manuscript

Härdle, W. K., Nasekin, S., Lee, D. K. C. and Phoon, K. F. (2014). TEDAS - Tail Event Driven ASset Allocation, submitted to Journal of Empirical Finance. 23.07.2015, manuscript ID, 15-239, SFB 649 DP 2015-32 .

Hartley, H. and Rao, J. (1967). Maximum-likelihood estimation for the mixed analysis of variance model, Biometrika 54(1-2): 93-108.

Huber, P. (1964). Robust Estimation of Location Parameter, The Annals of Mathematical Statistics 35(1): 73-101.

Hull, J. C. and White, A. D. (1998). Value at risk when daily changes in market variables are not normally distributed, The Journal of Derivatives 5(3): 9-19.

Jones, M. (1994). Expectiles and M-quantiles are quantiles, Statistics and Probability Letters 20(2): 149-153.

Koenker, R. (1993). When are expectiles percentiles?, Economic Theory 9(3): 526-527.

Koenker, R. (2005). Quantile regression, number 1, Econometric Society Monographs (Book 38), Cambridge university press.

Koenker, R. and Bassett, G. (1978). Regression quantiles, Econometrica 46(1).

Kusuoka, S. (2001). On law invariant coherent risk measures, Advances in mathematical economics : 83-95.

Laplace, P. (1793). Sur quelques points du systeme du monde, Memoires de l'Academie Royale des Sciences de Paris : 1-87. 
Mandelbrot, B. (1963). The variation of certain speculative prices, Journal of Business 36: $394-419$.

McNeil, A. and Frey, R. (2000). Estimation of tail-related risk measures for heteroscedastic financial time series: An extreme value approach, Journal of Empirical Finance 7: $271-300$.

Newey, W. K. and Powell, J. L. (1987). Asymmetric least squares estimation and testing, Econometrica 55(4).

Nolan, J. P. (2015). Stable Distributions - Models for Heavy Tailed Data, Birkhauser, Boston. In progress, Chapter 1 online at academic2.american.edu/ jpnolan.

Overbeck, L. (2004). Spectral capital allocation, RISK books, London .

Stahl, G., Zheng, J., Kiesel, R. and Rühlicke, R. (2012). Conceptualizing robustness in risk management, Available at SSRN 2065723.

Taylor, J. (2008). Estimating Value at Risk and Expected Shortfall Using Expectiles, Journal of Financial Econometrics 6(2): 231-252. 


\section{SFB 649 Discussion Paper Series 2015}

For a complete list of Discussion Papers published by the SFB 649, please visit http://sfb649.wiwi.hu-berlin.de.

001 "Pricing Kernel Modeling" by Denis Belomestny, Shujie Ma and Wolfgang Karl Härdle, January 2015.

002 "Estimating the Value of Urban Green Space: A hedonic Pricing Analysis of the Housing Market in Cologne, Germany" by Jens Kolbe and Henry Wüstemann, January 2015.

003 "Identifying Berlin's land value map using Adaptive Weights Smoothing" by Jens Kolbe, Rainer Schulz, Martin Wersing and Axel Werwatz, January 2015.

004 "Efficiency of Wind Power Production and its Determinants" by Simone Pieralli, Matthias Ritter and Martin Odening, January 2015.

005 "Distillation of News Flow into Analysis of Stock Reactions" by Junni L. Zhang, Wolfgang K. Härdle, Cathy Y. Chen and Elisabeth Bommes, January 2015.

006 "Cognitive Bubbles" by Ciril Bosch-Rosay, Thomas Meissnerz and Antoni Bosch-Domènech, February 2015.

007 "Stochastic Population Analysis: A Functional Data Approach" by Lei Fang and Wolfgang K. Härdle, February 2015.

008 "Nonparametric change-point analysis of volatility" by Markus Bibinger, Moritz Jirak and Mathias Vetter, February 2015.

009 "From Galloping Inflation to Price Stability in Steps: Israel 1985-2013" by Rafi Melnick and till Strohsal, February 2015.

010 "Estimation of NAIRU with Inflation Expectation Data" by Wei Cui, Wolfgang K. Härdle and Weining Wang, February 2015.

011 "Competitors In Merger Control: Shall They Be Merely Heard Or Also Listened To?" by Thomas Giebe and Miyu Lee, February 2015.

012 "The Impact of Credit Default Swap Trading on Loan Syndication" by Daniel Streitz, March 2015.

013 "Pitfalls and Perils of Financial Innovation: The Use of CDS by Corporate Bond Funds" by Tim Adam and Andre Guettler, March 2015.

014 "Generalized Exogenous Processes in DSGE: A Bayesian Approach" by Alexander Meyer-Gohde and Daniel Neuhoff, March 2015.

015 "Structural Vector Autoregressions with Heteroskedasticy" by Helmut Lütkepohl and Aleksei Netšunajev, March 2015.

016 "Testing Missing at Random using Instrumental Variables" by Christoph Breunig, March 2015.

017 "Loss Potential and Disclosures Related to Credit Derivatives - A CrossCountry Comparison of Corporate Bond Funds under U.S. and German Regulation" by Dominika Paula Gałkiewicz, March 2015.

018 "Manager Characteristics and Credit Derivative Use by U.S. Corporate Bond Funds" by Dominika Paula Gałkiewicz, March 2015.

019 "Measuring Connectedness of Euro Area Sovereign Risk" by Rebekka Gätjen Melanie Schienle, April 2015.

020 "Is There an Asymmetric Impact of Housing on Output?" by Tsung-Hsien Michael Lee and Wenjuan Chen, April 2015.

021 "Characterizing the Financial Cycle: Evidence from a Frequency Domain Analysis" by Till Strohsal, Christian R. Proaño and Jürgen Wolters, April 2015.

\section{SFB 649, Spandauer Straße 1, D-10178 Berlin http://sfb649.wiwi.hu-berlin.de}




\section{SFB 649 Discussion Paper Series 2015}

For a complete list of Discussion Papers published by the SFB 649, please visit http://sfb649.wiwi.hu-berlin.de.

022 "Risk Related Brain Regions Detected with 3D Image FPCA" by Ying Chen, Wolfgang K. Härdle, He Qiang and Piotr Majer, April 2015.

023 "An Adaptive Approach to Forecasting Three Key Macroeconomic Variables for Transitional China" by Linlin Niu, Xiu Xu and Ying Chen, April 2015.

024 "How Do Financial Cycles Interact? Evidence from the US and the UK" by Till Strohsal, Christian R. Proaño, Jürgen Wolters, April 2015.

025 "Employment Polarization and Immigrant Employment Opportunities" by Hanna Wielandt, April 2015.

026 "Forecasting volatility of wind power production" by Zhiwei Shen and Matthias Ritter, May 2015.

027 "The Information Content of Monetary Statistics for the Great Recession: Evidence from Germany" by Wenjuan Chen and Dieter Nautz, May 2015.

028 "The Time-Varying Degree of Inflation Expectations Anchoring" by Till Strohsal, Rafi Melnick and Dieter Nautz, May 2015.

029 "Change point and trend analyses of annual expectile curves of tropical storms" by P.Burdejova, W.K.Härdle, P.Kokoszka and Q.Xiong, May 2015.

030 "Testing for Identification in SVAR-GARCH Models" by Helmut Luetkepohl and George Milunovich, June 2015.

031 "Simultaneous likelihood-based bootstrap confidence sets for a large number of models" by Mayya Zhilova, June 2015.

032 "Government Bond Liquidity and Sovereign-Bank Interlinkages" by Sören Radde, Cristina Checherita-Westphal and Wei Cui, July 2015.

033 "Not Working at Work: Loafing, Unemployment and Labor Productivity" by Michael C. Burda, Katie Genadek and Daniel S. Hamermesh, July 2015.

034 "Factorisable Sparse Tail Event Curves" by Shih-Kang Chao, Wolfgang K. Härdle and Ming Yuan, July 2015.

035 "Price discovery in the markets for credit risk: A Markov switching approach" by Thomas Dimpfl and Franziska J. Peter, July 2015.

036 "Crowdfunding, demand uncertainty, and moral hazard - a mechanism design approach" by Roland Strausz, July 2015.

037 ""Buy-It-Now" or "Sell-It-Now" auctions : Effects of changing bargaining power in sequential trading mechanism" by Tim Grebe, Radosveta Ivanova-Stenzel and Sabine Kröger, August 2015.

038 "Conditional Systemic Risk with Penalized Copula" by Ostap Okhrin, Alexander Ristig, Jeffrey Sheen and Stefan Trück, August 2015.

039 "Dynamics of Real Per Capita GDP" by Daniel Neuhoff, August 2015.

040 "The Role of Shadow Banking in the Monetary Transmission Mechanism and the Business Cycle" by Falk Mazelis, August 2015.

041 "Forecasting the oil price using house prices" by Rainer Schulz and Martin Wersing, August 2015.

042 "Copula-Based Factor Model for Credit Risk Analysis" by Meng-Jou Lu, Cathy Yi-Hsuan Chen and Karl Wolfgang Härdle, August 2015.

043 "On the Long-run Neutrality of Demand Shocks" by Wenjuan Chen and Aleksei Netsunajev, August 2015.

\section{SFB 649, Spandauer Straße 1, D-10178 Berlin http://sfb649.wiwi.hu-berlin.de}




\section{SFB 649 Discussion Paper Series 2015}

For a complete list of Discussion Papers published by the SFB 649, please visit http://sfb649.wiwi.hu-berlin.de.

044 "The (De-)Anchoring of Inflation Expectations: New Evidence from the Euro Area" by Laura Pagenhardt, Dieter Nautz and Till Strohsal, September 2015.

045 "Tail Event Driven ASset allocation: evidence from equity and mutual funds' markets" by Wolfgang Karl Härdle, David Lee Kuo Chuen, Sergey Nasekin, Xinwen Ni and Alla Petukhina, September 2015.

046 "Site assessment, turbine selection, and local feed-in tariffs through the wind energy index" by Matthias Ritter and Lars Deckert, September 2015.

047 "TERES - Tail Event Risk Expectile based Shortfall" by Philipp Gschöpf, Wolfgang Karl Härdle and Andrija Mihoci, September 2015. 